\title{
Association of Massive Transfusion for Resuscitation in Gastrointestinal Bleeding with Transfusion-related Acute Lung Injury
}

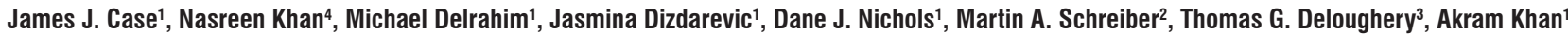 \\ ${ }^{1}$ Division of Pulmonary and Critical Care Medicine, Department of Medicine, ${ }^{2}$ Division of Trauma, Critical Care, and Acute Care Surgery, Department of Surgery, \\ ${ }^{3}$ Department of Hematology and Medical Oncology, School of Medicine, Oregon Health and Science University, Portland, OR, ${ }^{4}$ Real World Economics Consultancy, \\ Mendham, NJ, USA
}

\section{Abstract}

Background and Aims: This study aimed to understand the use of massive transfusion (MT) for gastrointestinal bleeding (GIB). Patients and Methods: We performed a retrospective analysis of patients admitted to our medical Intensive Care Unit (ICU) with GIB for the type of bleeding, quantity of blood products transfused, and risk of transfusion-related acute lung injury (TRALI) and death. MT was defined as transfusion of 10 or more units of red blood cell (RBC) within a 24-h period in a 1-unit RBC: 1-unit fresh frozen plasma: and 1-unit platelet ratio. TRALI was defined as development of acute lung injury (ALI), within $6 \mathrm{~h}$ of transfusion, with new bilateral pulmonary infiltrates, absence of circulatory overload, or other explanation for ALI. Results: In a 43-month interval, 169 patients were admitted to the ICU with GIB and received blood products, of whom 13 received MT. Ten patients developed TRALI, of whom 7 (70\%) had received MT. MT was associated with an increased risk of TRALI (odds ratio [OR]: 17.9, 95\% confidence interval [CI]: 2.9-111.2, $P=0.002$ ) after adjusting for age, sex, body mass index, baseline vitals, and laboratory data. Death was predicted by MT (OR: 5.6, 95\% CI: 1.6-19.7, $P=0.007)$, TRALI (OR: 2.3, 95\% CI: 1.1-4.6, $P=0.02$ ), and Acute Physiologic Chronic Health Evaluation II score (OR: 1.17 per unit increase, $95 \%$ CI: $1.09-1.26, P<0.001)$ after adjusting for age and sex. Conclusions: MT for GIB is associated with an increased risk of TRALI and death. Prospective studies assessing the use of MT in this population are needed to understand and improve outcomes.

Keywords: Blood transfusion, gastrointestinal bleeding, Intensive Care Unit, massive transfusion, outcomes, resuscitation

\section{INTRODUCTION}

Massive transfusion (MT) of blood products has been associated with multiple organ failure, systemic inflammatory response syndrome, transfusion-related acute lung injury (TRALI), transfusion associated circulatory overload (TACO), increased infection, and increased mortality. ${ }^{[1,2]}$

TRALI is characterized by dyspnea, hypoxemia, and acute interstitial pulmonary infiltrates within $6 \mathrm{~h}$ of blood product administration in the absence of other precipitating factors. ${ }^{[2,3]}$ TRALI, while associated with the administration of all forms of blood products, has most commonly been described following the administration of fresh frozen plasma (FFP), especially in patients with preexisting hepatic disease. ${ }^{[4]}$ Studies have assessed the effect of MTs on the development of TRALI in trauma patients, but there is limited data in medical patients. ${ }^{[4-10]}$

\begin{tabular}{|l|l|}
\hline \multicolumn{3}{c|}{ Access this article online } \\
\hline Quick Response Code: & Website: \\
& www.ijccm.org \\
& \\
\end{tabular}

This study aimed to examine factors that lead to the use of MT in patients with gastrointestinal bleeding (GIB) in a medical Intensive Care Unit (ICU) as well as complications associated with the use of MT in this population.

\section{Materials and Methods}

\section{Patient selection}

After obtaining approval from our Institutional Review Board, we queried the hospital database for all patients who

Address for correspondence: Dr. Akram Khan, Oregon Health and Science University, 3181 SW Sam Jackson Park Road, UHN67, Portland, OR, USA. E-mail: khana@ohsu.edu

This is an open access article distributed under the terms of the Creative Commons Attribution-NonCommercial-ShareAlike 3.0 License, which allows others to remix, tweak, and build upon the work non-commercially, as long as the author is credited and the new creations are licensed under the identical terms.

For reprints contact: reprints@medknow.com

How to cite this article: Case JJ, Khan N, Delrahim M, Dizdarevic J, Nichols DJ, Schreiber MA, et al. Association of massive transfusion for resuscitation in gastrointestinal bleeding with transfusion-related acute lung injury. Indian J Crit Care Med 2017;21:506-13. 
had a discharge diagnosis of GIB based on the International Classification of Diseases-9 (ICD-9) code 578.x between April 1, 2008, and October 31, 2011. This was cross-referenced with a database of all blood product transfusions during the same time. The list of matching patients was screened for admissions to the ICU, either directly from the emergency room/outside facility or through inpatient transfers from medicine floors. The records of patients who met these criteria were then analyzed for data regarding demographics, blood product administration (timing and product type), duration of admission to the ICU and hospital, and risk of death during the first 28 days, Model for End-Sage Liver Disease (MELD) score, and Acute Physiologic Chronic Health Evaluation (APACHE) II score. We also obtained data regarding diagnostic testing to identify the location and etiology of the GIB.

The standard practices at our institution are to admit or transfer patients with active GIB to the medical ICU service, use leukoreduced red blood cells (RBCs) and apheresed platelets (PLT) equivalent to 6 units of nondonor-matched PLT. We also have a 95\% male-only FFP policy with only Rh type $\mathrm{AB}$ plasma having the possibility of female donors. To standardize data, vital signs and laboratory values on ICU admission were used for data analysis except in situations where the most abnormal value was preferred (e.g., APACHE II calculations). Our institution uses a standard transfusion threshold of hemoglobin $<7 \mathrm{~g} / \mathrm{dL}$ for transfusion except in cases of hemodynamic instability and transfusion of 10 or more units of $\mathrm{RBC}$ within a 24-h period in a 1 unit RBC: 1 unit FFP: 1 unit PLT ratio as MT.

\section{Outcomes}

MT was defined as transfusion of 10 or more units of RBC within a 24-h period in a 1 unit RBC: 1 unit FFP: 1 unit PLT ratio. ${ }^{[1-13]}$ ICU vital signs were monitored for worsening Alveolar-arterial gradient with an increase in the fraction of inspired oxygen $\left(\mathrm{FiO}_{2}\right)$ needed to maintain an oxygen saturation $\left(\mathrm{SpO}_{2}\right) \geq 92 \%$ and $\mathrm{PaO}_{2} \geq 55 \mathrm{mmHg}$.

TRALI has been previously defined as development of new acute lung injury (ALI) with onset of signs and symptoms within $6 \mathrm{~h}$ of transfusion with new bilateral pulmonary infiltrates, absence of circulatory overload, or preexisting ALI before transfusion with no other ALI risk factors such as pneumonia or aspiration. ${ }^{[2,3]} \mathrm{A} \mathrm{PaO}_{2} / \mathrm{FiO}_{2}$ ratio $\leq 300$ was used as the definition of ALI. ${ }^{[14]}$ If no arterial blood gas sampling was performed, change in saturation to $\mathrm{FiO}_{2}$ (SF ratio) from before transfusion was used. ${ }^{[15-19]}$ All cases of ALI were assessed for other causes of lung injury including sepsis, pneumonia, aspiration, or severe pancreatitis which had to be ruled out to qualify for a TRALI designation. Review of radiology films along with the official radiology interpretation was used for determination of bilateral infiltrates. Two authors (JC, MD) reviewed all cases with adjudication by the senior author (AK) in case of issues.

Microbiology results were reviewed for infection defined as a sputum culture with a known bacterial pathogen, urinary culture with $>100,000$ colony-forming units of a known pathogen, or a blood culture positive for a bacterial or fungal pathogen. For patients with prolonged hospital admissions who may have required frequent transfusions (bone marrow transplant patients, hematologic malignancy, severe myelodysplastic syndrome, etc.), only transfusion data during the ICU admission were used in the analysis. MELD and APACHE II scores were calculated using the published guidelines..$^{[20,21]}$

We used 28-day ICU-free days to avoid the confounding effect of death during hospitalization. ${ }^{[22]}$ ICU-free days in a 28-day period were determined by subtracting the ICU length of stay (LOS) from 28. ${ }^{[22,23]}$ The 28-day ICU-free days were considered to be zero if a patient died before ICU discharge or stayed in the ICU for longer than 28 days.

\section{Statistical analysis}

Baseline demographic and clinical characteristics of patients stratified by massive versus non-MT are summarized in Table 1 with categorical variables presented as counts or frequencies and continuous data summarized by median and interquartile range (IQR). For each characteristic, a Wilcoxon rank-sum test or Chi-square test was carried out to determine statistical differences.

To identify the demographic and clinical characteristics associated with TRALI and risk of death, a step-wise regression procedure was used. A significance level of 0.05 was used as the cutoff for adding variables to the model, and a level of 0.20 was used as the cutoff for removing variables. For model development, based on significance on univariate analysis, APACHE II score, MELD score, mean arterial pressure (MAP), hematocrit, and respiratory rate were locked in the model for TRALI. APACHE II score, MELD score, and TRALI were locked in the model for death. All analyses were carried out in Stata IC version 13.1 (StataCorp LP, College Station, TX, USA).

\section{RESULTS}

During the 43 months between April 1, 2008, and October $31,2011,1168$ patients had a discharge diagnosis of GIB and $214(18.3 \%)$ received at least one transfusion. $169(79 \%)$ of these patients were admitted to the ICU and were included in the final data analysis. During the same period, there were 2154 admissions to the ICU, of whom 1544 (71.7\%) were discharged from the hospital while 610 (28.3\%) died during the hospital admission [Figure 1]. There were $901(41.8 \%)$ females and $1253(58.2 \%)$ males. The average age of the patients admitted to the ICU was $54.0 \pm 16.5$ years. Average LOS in the ICU was $11.0 \pm 14.1$ days. The mean number of ICU-free days was $13.4 \pm 11.8$. The 169 patients with GIB constituted $7.9 \%$ of ICU admissions.

The median age of patients with GIB was 61 years, (IQR: $51-$ 69 ), the composition of this population was $42 \%$ of females and $58 \%$ of males, the median LOS in the hospital was 6 days (IQR: $3-13$ ), and the median LOS in the ICU was 3 days (IQR: 
Table 1: Differences in demographics, admission vitals, length of stay, use of blood products, and outcomes in patients with gastrointestinal bleeding with and without the use of massive transfusion

\begin{tabular}{|c|c|c|c|}
\hline & \multicolumn{2}{|c|}{ IQR } & \multirow[t]{2}{*}{ Wilcoxon rank-sum $/ \chi^{2}(P)$} \\
\hline & MT median (25\%-75\%) & Non-MT median (25\%-75\%) & \\
\hline \multicolumn{4}{|l|}{ Demographics } \\
\hline$n(\%)$ & $13(7.7)$ & $156(92.3)$ & \\
\hline Age (years) & $61(52-68)$ & $61(51-69.5)$ & 0.9506 \\
\hline BMI $\left(\mathrm{kg} / \mathrm{m}^{2}\right)$ & $28.88(26.45-32.82)$ & $26.91(22.59-32.28)$ & 0.6018 \\
\hline \multicolumn{4}{|l|}{ Admission vitals } \\
\hline Heart rate (beats/min) & $117(86-151)$ & $108(70-124)$ & 0.2410 \\
\hline Respiratory rate (breaths/min) & $26(12-30)$ & $25(16-30)$ & 0.7903 \\
\hline MAP* (mmHg) & $51(47-55)$ & $57(48-66)$ & 0.0114 \\
\hline Temperature $\left({ }^{\circ} \mathrm{C}\right)$ & $36.3(35.7-36.7)$ & $36.55(36.1-37)$ & 0.1297 \\
\hline Glasgow Coma Scale* & $3(3-13)$ & $15(11-15)$ & 0.0001 \\
\hline \multicolumn{4}{|l|}{ Admission laboratory data } \\
\hline Serum creatinine $(\mathrm{mg} / \mathrm{dL})$ & $1.29(0.98-1.72)$ & $1.045(0.8-1.68)$ & 0.5631 \\
\hline Serum sodium $(\mathrm{mEq} / \mathrm{L})$ & $137(130-141)$ & $137(133.5-141)$ & 0.5823 \\
\hline Serum potassium $(\mathrm{mEq} / \mathrm{L})$ & $4(3.8-5.7)$ & $4.2(3.8-4.8)$ & 0.9013 \\
\hline Serum bicarbonate $(\mathrm{mmol} / \mathrm{L})$ & $21(14-26)$ & $23(19-25)$ & 0.2317 \\
\hline Serum AST (SGOT) $(\mathrm{IU} / \mathrm{L})^{*}$ & $129(63-909)$ & $45(28-103)$ & 0.0030 \\
\hline Serum ALT (SGPT)* (IU/L) & $96(33-336)$ & $35(20-71)$ & 0.0275 \\
\hline Serum total bilirubin* (mg/dL) & $2.6(1.1-5.3)$ & $1.3(0.8-2.7)$ & 0.0316 \\
\hline HCT (\%) & $20.4(15.6-24)$ & $24.65(22.25-27.8)$ & 0.0286 \\
\hline $\mathrm{INR}^{*}$ & $2.4(2.04-3.04)$ & $1.475(1.21-1.815)$ & 0.0002 \\
\hline APACHE II* & $32(24-37)$ & $19(14-27)$ & 0.0009 \\
\hline MELD score* & $25(22-29)$ & $15(10-21)$ & 0.0023 \\
\hline \multicolumn{4}{|l|}{ LOS (days) } \\
\hline Total LOS & $8(2-11)$ & $6(3.5-13)$ & 0.6636 \\
\hline ICU LOS & $5(2-6)$ & $3(2-6)$ & 0.8648 \\
\hline ICU-free days* & $0(0-0)$ & $20(0-25)$ & 0.0003 \\
\hline \multicolumn{4}{|l|}{ Blood products } \\
\hline $\operatorname{RBC}\left(1^{\text {st }} 24 \mathrm{~h}\right)^{*, \#}$ & $10(9-14)$ & $2(1.5-4)$ & 0.0000 \\
\hline RBC (total ICU stay)* & $11(9-14)$ & $3(2-6)$ & $<0.0001$ \\
\hline $\operatorname{FFP}\left(1^{\text {st }} 24 \mathrm{~h}\right)^{*}$ & $11(9-28)$ & $0(0-2)$ & $<0.0001$ \\
\hline FFP (total ICU stay)* & $16(9-30)$ & $0(0-4)$ & $<0.0001$ \\
\hline $\operatorname{PLT}\left(1^{\text {st }} 24 \mathrm{~h}\right)^{*}$ & $18(12-36)$ & $0(0-6)$ & $<0.0001$ \\
\hline PLT (total ICU stay)* & $18(12-48)$ & $0(0-12)$ & 0.0002 \\
\hline $\mathrm{CP}\left(1^{\text {st }} 24 \mathrm{~h}\right)^{*}$ & $0(0-2)$ & $0(0-0)$ & $<0.0001$ \\
\hline CP (total ICU stay)* & $0(0-2)$ & $0(0-0)$ & $<0.0001$ \\
\hline \multicolumn{4}{|l|}{ Outcomes } \\
\hline TRALI* & 6 & 4 & $<0.0001$ \\
\hline Alive* & 4 & 108 & 0.011 \\
\hline Dead & 9 & 48 & \\
\hline
\end{tabular}

*Statistically significant difference, ${ }^{\#}$ In the MT group, 3 patients received 2 units and one patient received 1 unit of RBC before transfer to our facility, those units were not included in data analysis. AST: Aspartate aminotransferase; ALT: Alanine aminotransferase; INR: International normalized ratio; APACHE: Acute Physiologic Chronic Health Evaluation; MELD: Model for End-Stage Liver Disease; ICU: Intensive Care Unit; RBC: Red blood cell; FFP: Fresh frozen plasma; PLT: Platelets; CP: Cryoprecipitate; TRALI: Transfusion-related acute lung injury; IQR: Interquartile range; LOS: Length of stay; MAP: Mean arterial pressure; SGOT: Serum glutamic-oxaloacetic transaminase; SGPT: Serum glutamic pyruvic transaminase; BMI: Body mass index; HCT: Hematocrit; MT: Massive transfusion

2-6). Nearly $66.3 \%$ of patients were alive and discharged from the hospital while $33.7 \%$ died during the hospital stay. The median number of RBC units transfused in the first $24 \mathrm{~h}$ was 2 units [IQR: 2-4, Figure 2], and the median number of RBC units transfused in the entire stay was 4 units [IQR: $2-8$, Figure 3]. In the MT group, three patients received 2 units and one patient received 1-unit RBC before transfer to our facility.
The MT protocol was used in $13(7.7 \%)$ patients with GIB. Eight patients had bleeding caused by a source proximal to the pylorus based on clinical findings of hematemesis (one patient with bleeding after percutaneous endoscopic gastrostomy placement), one had bleeding from a colon rupture, and the source of bleeding could not be identified in four patients due to hemodynamic instability precluding 


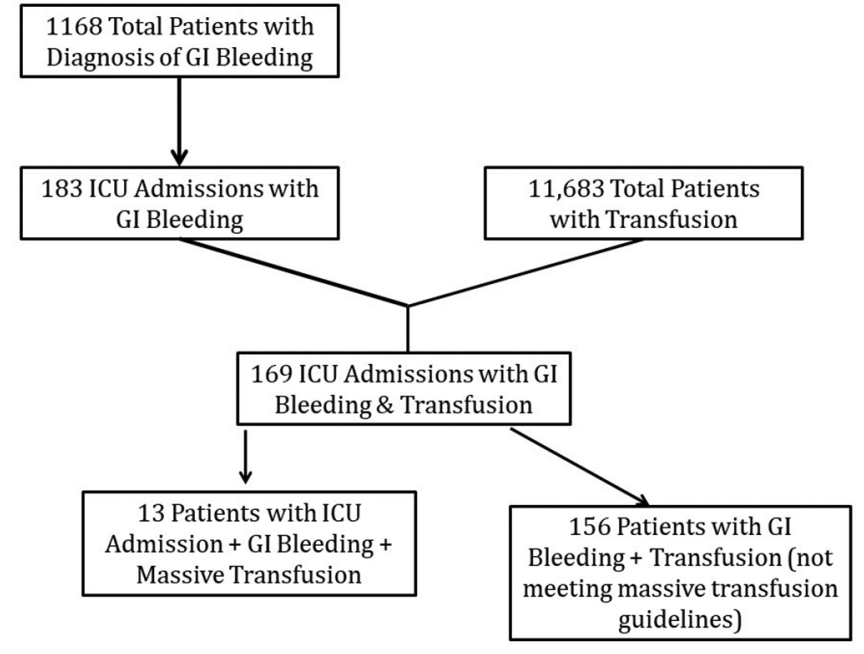

Figure 1: Patient selection flow sheet

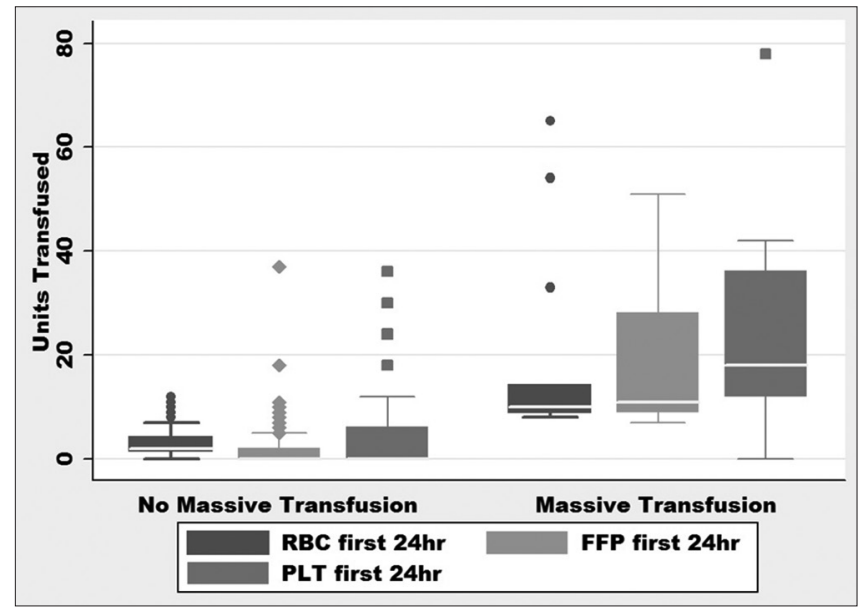

Figure 2: Units of blood products transfused in $1^{\text {st }} 24 \mathrm{~h}$ of presentation. RBC: Red blood cell, PLT: Platelet, FFP: Fresh frozen plasma

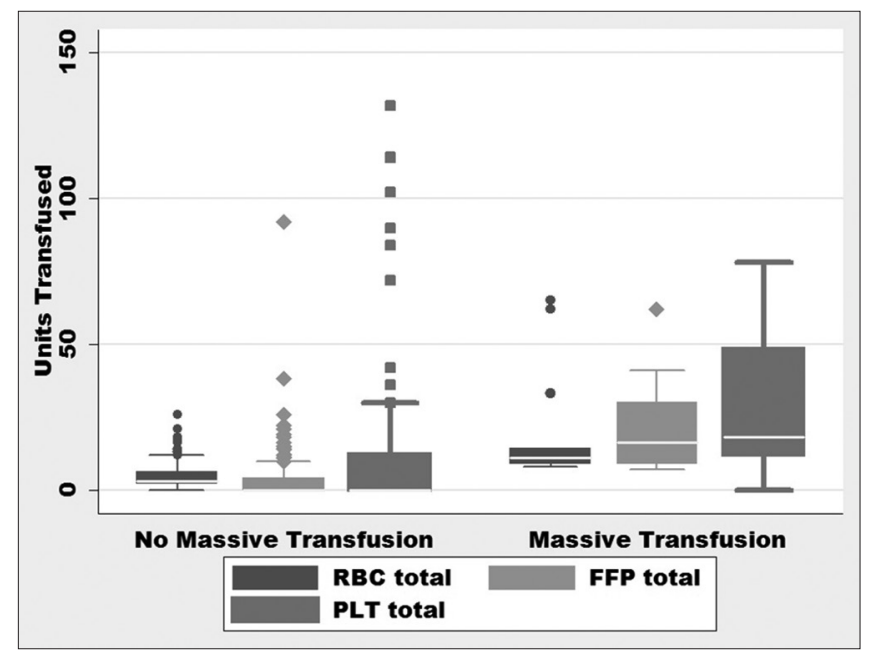

Figure 3: Units of blood products transfused during the entire hospitalization. RBC: Red blood cell, PLT: Platelet, FFP: Fresh frozen plasma

further diagnostic testing. The diagnostic testing and source of bleeding for patients receiving MT are listed in Table 2.

\begin{tabular}{lll}
\hline \multicolumn{3}{l}{ Table 2: Diagnostic testing and source of bleeding in } \\
\multicolumn{1}{l}{$\mathbf{l}$ patients requiring massive transfusion } \\
\hline Patient & $\begin{array}{l}\text { Diagnostic } \\
\text { testing }\end{array}$ & Bleeding source \\
\hline 1 & None & Unknown \\
2 & EGD & Unknown \\
3 & EGD & Esophageal varices, portal hypertensive \\
& & gastropathy, and Mallory-Weiss tear \\
4 & None & Unknown \\
5 & None & Unknown \\
6 & EGD & Varices \\
7 & EGD & Aortoesophageal fistula \\
8 & None & Unknown \\
9 & None & Gastro-aortic fistula \\
10 & None & Unknown \\
11 & None & Unknown \\
12 & EGD & Postoperative PEG bleeding \\
13 & None & Colon rupture \\
\hline
\end{tabular}

EGD: Esophagogastroduodenoscopy; PEG: Percutaneous endoscopic gastrostomy

The median number of units transfused (RBC: FFP: PLT) in the MT group was 10:11:18 compared to 2:0:0 in the non-MT group, with ratios of 1:1:1.8 versus 1:0:0 in the two groups, respectively $(P<0.03)$ [Figures 2 and 3]. Of the 13 patients who received MT, 4 had a diagnosis of cirrhosis or chronic liver disease with a MELD of 26.75 (range: 22-33), the remaining patients had a MELD of 23.2 (range: 10-37). No patients received a prothrombin complex concentrate or tranexamic acid to control bleeding. After adjusting for age, sex, and weight, initiation of MT increased the odds ratio $(\mathrm{OR})$ of death by 5.6 (95\% confidence interval $[\mathrm{CI}]$ : 1.6-19.7, $P=0.007)$.

TRALI developed in $10(5.9 \%)$ of the 169 patients [Table 1 ]. Six $(46.2 \%)$ of the 13 patients who received MT developed TRALI, by contrast $4(2.6 \%)$ of the 156 patients who did not require MT developed TRALI. Development of TRALI increased OR for death for 2.3 times (95\% CI: 1.1-4.6, $P=0.02)$. Infections developed in $16(9.5 \%)$ patients, only 1 of whom required MT. The median ICU LOS was 5 days (IQR: 2-6) for patients who received MT and 3 days (IQR: 2-6) for those who did not receive MT $(P=0.86)$. Patients who received MT had no ICU-free days in a 28-day period, while the contrasting population had 20 ICU-free days (IQR: $0-25, P=0.0003$ ). The number of units transfused in the first $24 \mathrm{~h}$ as well as total units transfused for the admission was higher in the TRALI group [Table 1]. Evaluation of admission vital signs showed that MAP at admission was lower in individuals who required $\mathrm{MT}$, $51 \mathrm{mmHg}$ (IQR: 47-55) compared to $57 \mathrm{mmHg}$ [IQR: 48-66, $P=0.0114$, Table 1] in individuals who did not. The need for MT could not be predicted by any baseline laboratory values including admission hematocrit. Nine out of 13 patients $(69.2 \%)$ who received MT died compared to 48 out of 156 patients $(30.8 \%)$ who did not with OR for 
death at $5.06(95 \% \mathrm{CI}: 1.32-23.37, P=0.0048)$ for those who received MT. In scenarios where the site of bleeding could not be identified, it was more likely that MT was used as a treatment. Of the 13 patients without a known source of bleeding, 6 (46.2) required activation of the MT protocol. TRALI did not increase the duration of ICU stay or the total hospital stay but was associated with reduced ICU-free days [Table 3].

In a step-wise regression procedure with forward selection, after adjusting for age, sex, body mass index (BMI), baseline vitals, and laboratory data, MT was associated with an increased risk of TRALI with OR 17.9 [95\% CI: 2.9-111.2, $P=0.002$, Table 4]. After adjusting for age and sex, death was predicted by the APACHE II score with an increase in OR 1.17 per unit increase in APACHE II score (95\% CI: 1.09-1.26 $P<0.001)$; baseline hematocrit, OR 1.0 (95\% CI: 1.01-1.18, $P=0.027)$; admission respiratory rate, OR $1.06(95 \% \mathrm{CI}$ : 1.01-1.11, $P=0.023)$; and BMI, OR 1.11 (95\% CI: 1.04-1.17, $P=0.001)$, but not by the use of MT protocol or development of TRALI [Table 5].

Table 3: Differences between patients who developed transfusion-related acute lung injury and those who did not

\begin{tabular}{|c|c|c|c|}
\hline & \multicolumn{2}{|c|}{ IQR } & \multirow[t]{2}{*}{ Wilcoxon test $/ \chi^{2}(P)$} \\
\hline & TRALI median (25\%-75\%) & Non-TRALI (25\%-75\%) & \\
\hline \multicolumn{4}{|l|}{ Demographics } \\
\hline$n(\%)$ & $10(5.9)$ & $159(94.1)$ & \\
\hline Age (years) & $63(49-73)$ & $61(51-69)$ & 0.94 \\
\hline BMI $\left(\mathrm{kg} / \mathrm{m}^{2}\right)$ & $28.8(22-32.5)$ & $27(23.1-32.3)$ & 0.90 \\
\hline \multicolumn{4}{|l|}{ Admission vitals } \\
\hline Heart rate (beats/min) & $120.5(62-151)$ & $109(70-124)$ & 0.49 \\
\hline Respiratory rate (breaths/min) & $29(25-39)$ & $25(12-30)$ & 0.94 \\
\hline MAP* (mmHg) & $51.5(49-55)$ & $57(48-66)$ & 0.19 \\
\hline Temperature $\left({ }^{\circ} \mathrm{C}\right)$ & $36.4(36-36.9)$ & $36.5(36.1-37)$ & 0.63 \\
\hline Glasgow Coma Scale* & $4.5(3-15)$ & $15(10-15)$ & 0.035 \\
\hline \multicolumn{4}{|l|}{ Admission laboratory data } \\
\hline Serum creatinine $(\mathrm{mg} / \mathrm{dL})$ & $1.33(1.06-1.9)$ & $1.04(0.8-1.65)$ & 0.083 \\
\hline Serum sodium $(\mathrm{mEq} / \mathrm{L})$ & $136(131-140)$ & $137(133-141)$ & 0.46 \\
\hline Serum potassium $(\mathrm{mEq} / \mathrm{L})$ & $4.35(3.9-6.6)$ & $4.2(3.8-4.8)$ & 0.368 \\
\hline Serum bicarbonate $(\mathrm{mmol} / \mathrm{L})$ & $21(14-26)$ & $23(19-25)$ & 0.2317 \\
\hline Serum AST (SGOT)* (IU/L) & $96.5(61-287)$ & $45.5(28-104)$ & 0.023 \\
\hline Serum ALT (SGPT) (IU/L) & $66.5(23-112)$ & $35.5(20-72)$ & 0.164 \\
\hline Serum total bilirubin $(\mathrm{mg} / \mathrm{dL})$ & $2.55(1.1-5.3)$ & $1.3(0.8-2.7)$ & 0.1537 \\
\hline $\mathrm{HCT}^{*}(\%)$ & $20.65(15.6-22.3)$ & $24.7(22.2-27.8)$ & 0.0029 \\
\hline INR* & $2.36(1.63-2.74)$ & $1.5(1.21-1.9)$ & 0.027 \\
\hline APACHE II* & $34(20-37)$ & $19(14-27)$ & 0.007 \\
\hline MELD score* & $27.5(13-29)$ & $15(11-22)$ & 0.056 \\
\hline \multicolumn{4}{|l|}{ LOS (days) } \\
\hline Total LOS & $8.5(4-20)$ & $6(3-13)$ & 0.496 \\
\hline ICU LOS & $6.5(4-9)$ & $3(2-6)$ & 0.078 \\
\hline ICU-free days* & $0(0-10)$ & $19.5(0-25)$ & 0.018 \\
\hline \multicolumn{4}{|l|}{ Blood products } \\
\hline $\operatorname{RBC}\left(1^{\text {st }} 24 \mathrm{~h}\right)^{*, \#}$ & $8(4-12)$ & $2(2-4)$ & 0.0003 \\
\hline RBC (total ICU stay)* & $8(12-18)$ & $3(2-7)$ & 0.0001 \\
\hline $\operatorname{FFP}\left(1^{\text {st }} 24 \mathrm{~h}\right)^{*}$ & $8.5(6-11)$ & $0(0-2)$ & 0.0001 \\
\hline FFP (total ICU stay)* & $13.5(9-21)$ & $0(0-4)$ & $<0.0001$ \\
\hline $\operatorname{PLT}\left(1^{\text {st }} 24 \mathrm{~h}\right)^{*}$ & $9(0-24)$ & $0(0-6)$ & $<0.0001$ \\
\hline PLT (total ICU stay)* & $15(6-30)$ & $0(0-18)$ & 0.0083 \\
\hline $\mathrm{CP}\left(1^{\text {st }} 24 \mathrm{~h}\right)^{*}$ & $0(0-2)$ & $0(0-0)$ & 0.0112 \\
\hline $\mathrm{CP}$ (total ICU stay)* & $0(0-2)$ & $0(0-0)$ & 0.0026 \\
\hline \multicolumn{4}{|l|}{ Outcomes } \\
\hline Dead* & 7 & 50 & 0.032 \\
\hline Alive & 3 & 109 & \\
\hline
\end{tabular}

*Statistically significant difference, ${ }^{\text {In }}$ the massive transfusion group 3 subjects received 2 units and one subject 1 unit of RBC before transfer to our facility, those units were not included in data analysis. AST: Aspartate aminotransferase; ALT: Alanine aminotransferase; INR: International normalized ratio; APACHE: Acute Physiologic and Chronic Health Evaluation; MELD: Model for End-Stage Liver Disease; ICU: Intensive Care Unit; RBC: Red blood cell; FFP: Fresh frozen plasma; PLT: Platelets; CP: Cryoprecipitate; TRALI: Transfusion-related acute lung injury; IQR: Interquartile range; LOS: Length of stay; MAP: Mean arterial pressure; SGOT: Serum glutamic-oxaloacetic transaminase; SGPT: Serum glutamic pyruvic transaminase; BMI: Body mass index; HCT: Hematocrit 


\section{Discussion}

TRALI represents $37 \%$ of transfusion-related fatalities. ${ }^{[24]}$ Data on the incidence of TRALI while using MT in medical patients with GIB are limited.$^{[5,25]}$ We performed a retrospective analysis of patients who received transfusions for GIB to determine the association between TRALI and use of MT [Tables 1 and 3]. After adjusting for age, sex, and BMI, MT use was associated with an increased risk of developing TRALI while the baseline APACHE II and MELD scores were not suggesting that TRALI was associated with the number of units transfused rather than the underlying severity of illness or presence of liver disease [Table 4]. Odds of death were increased with TRALI on univariate analysis, but after adjusting for age and sex, death was predicted by APACHE II score, admission hematocrit, admission respiratory rate, and BMI [Table 5].

Contrary to other studies, after adjusting for age and sex, our study did not show an increase in the incidence of death with increasing MELD score. ${ }^{[20,26,27]}$ These differences may be

\begin{tabular}{lcccc}
\hline $\begin{array}{l}\text { Table 4: Model for association between } \\
\text { transfusion-related acute lung injury and use of massive } \\
\text { transfusion protocol }\end{array}$ & $\mathbf{O R}$ & $\mathbf{Z}$ & $\boldsymbol{P}>\mathbf{~ Z |}$ & $\mathbf{9 5 \%} \mathbf{~ C l}$ \\
\hline TRALI odds & 0.996 & -0.1 & 0.93 & $0.9-1.1$ \\
\hline APACHE II & 1.1 & 1.2 & 0.25 & $0.97-1.13$ \\
MELD score & 0.99 & -0.3 & 0.77 & $0.94-1.05$ \\
MAP at admission & 0.91 & -1.4 & 0.16 & $0.79-1.04$ \\
HCT & 1.1 & 1.8 & 0.069 & $0.99-1.19$ \\
Respiratory rate at admission & 17.9 & 3.1 & 0.002 & $2.9-111.2$ \\
MT protocol &
\end{tabular}

For model development purposes, based on significance and univariate analysis, APACHE II score, MELD score, mean arterial pressure, HCT, and respiratory rate were locked in the model for TRALI. APACHE: Acute Physiologic and Chronic Health Evaluation; MELD: Model for End-Stage Liver Disease; TRALI: Transfusion-related acute lung injury; MAP: Mean arterial pressure; HCT: Hematocrit; MT: Massive transfusion; OR: Odds ratio; CI: Confidence interval

\begin{tabular}{lcccc}
\hline $\begin{array}{l}\text { Table 5: Model for association between death and use of } \\
\text { massive transfusion protocol }\end{array}$ \\
\hline Death & $\mathbf{O R}$ & $\mathbf{Z}$ & $\boldsymbol{P}>\mathbf{Z}$ & $\mathbf{9 5 \%} \mathbf{~ C l}$ \\
\hline APACHE II & 1.17 & 4.3 & $<0.001$ & $1.09-1.26$ \\
TRALI & 2.63 & 0.96 & 0.339 & $0.363-19.03$ \\
MELD Score & 0.97 & -0.5 & 0.605 & $0.94-1.04$ \\
MAP & 1.01 & 1.3 & 0.188 & $0.99-1.04$ \\
HCT & 1.09 & 2.2 & 0.027 & $1.01-1.18$ \\
Admission respiratory rate & 1.06 & 2.3 & 0.023 & $1.01-1.11$ \\
MT protocol & 3.17 & 1.4 & 0.17 & $0.61-16.52$ \\
BMI & 1.11 & 3.2 & 0.001 & $1.04-1.17$ \\
\hline
\end{tabular}

For model development purposes, based on significance and univariate analysis, APACHE II score, MELD score, and TRALI were locked in the model for death. APACHE: Acute Physiologic and Chronic Health Evaluation; MELD: Model for End-Stage Liver Disease; TRALI: Transfusion-related acute lung injury; MAP: Mean arterial pressure; HCT: Hematocrit; MT: Massive transfusion; OR: Odds ratio; CI: Confidence interval; BMI: Body mass index explained either by our patients being transfused in a 1:1:1.8 ratio of RBC: FFP: PLT, thereby correcting coagulation abnormalities which may have mitigated the effects of abnormal liver function, or by the low incidence of severe liver disease in our patients.

The ratios of blood products transfused in the MT group in our study of 1:1:1.8 was consistent with the current MT guidelines of 1:1:1 as an approximation of whole blood. This is similar to the Pragmatic, Randomized Optimal Platelets and Plasma Ratios study which compared 1:1:1-1:1:2 transfusion ratios in trauma patients and showed that early administration of plasma, PLT, and RBCs in a 1:1:1 ratio compared with a 1:1:2 ratio did not result in significant differences in the risk of death within the first $24 \mathrm{~h}$ or at 30 days. ${ }^{[28]}$

Our cohort had an increased risk of developing TRALI with the use of all blood products including FFP. Studies have noted the associations of FFP administration, especially female FFP, with TRALI ${ }^{[25]}$ Our institution uses male plasma for $95 \%$ of all FFP units other than AB blood type to decrease this likelihood. Our sample size was too small to assess for a dose-dependent association of blood products with TRALI due to the increased possibility of type II error.

Our study showed a slight increase in the risk of death with increasing BMI. Data describing the effects of BMI on ICU mortality are conflicting, but the general trend shows an inverse relationship between BMI and mortality. ${ }^{[29-33]}$ Often, a higher BMI is protective against mortality ${ }^{[33]}$ but we did not find that in our study.

After adjusting for age, sex, and BMI, we were not able to identify any clinical parameters that could predict the need for utilization of a MT protocol. We noted that the use of MT was more likely when the site of bleeding could not be identified. This may be due to the fact that hemodynamically unstable patients are often incapable of enduring diagnostic procedures to help identify the exact source of bleeding.

The retrospective nature of our study makes it difficult to differentiate between TRALI, acute respiratory distress syndrome (ARDS) or transfusion-associated cardiac overload (TACO). We took all possible measures to exclude patients with ARDS and TACO from our TRALI group. We used the expert panel criteria for adjudication of acute posttransfusion hypoxemia with bilateral pulmonary infiltrates to differentiate between TRALI, TACO, ARDS/ALI, or other etiologies such as pneumonia, aspiration, congestive heart failure, or diffuse pulmonary hemorrhage by chart review. Initial screening was done by two authors followed by adjudication by the senior author to minimize the risk of inaccuracy. ${ }^{[3,14]}$ The hypovolemic nature of patients receiving MT for GI bleeding from hemorrhagic shock also reduces the chances of patients developing volume overload. ${ }^{[34]}$ As our study is retrospective, we were unable to establish a causal link between MT and the development of TRALI. Prospective studies are needed to clarify this association. Based on ICD-9 data, we identified 
that it is possible that some patients with GIB may have been missed due to incorrect coding. Finally, these data represent information from only a single, academic tertiary center ICU and may not be suitable for extrapolation to all practice environments.

Despite these limitations, our study is one of the few assessing the role of MT in medical patients with GI bleeding and provides useful insight into risk factors for TRALI and the risk of death in this population.

\section{Conclusion}

In this retrospective analysis, we found that GIB constituted $7.9 \%$ of medical ICU admissions. A small percentage of these patients $(7.7 \%)$ required activation of MT. The incidence of TRALI increased with the activation of MT as part of the resuscitation. After adjusting for age, sex, and $\mathrm{BMI}$, the risk of death increased with the initiation of $\mathrm{MT}$, and MT was associated with decreased 28-day ICU-free survival. Death was predicted by the severity of illness as determined by the APACHE II score, baseline hematocrit, admission respiratory rate, and BMI. Prospective studies of MT in medical patients with GIB may be helpful in determining risk factors for TRALI and risk factors which increase mortality.

\section{Financial support and sponsorship}

This publication was made possible with support from the Oregon Clinical and Translational Research Institute, grant number UL1 RR024140, from the National Center for Advancing Translational Sciences, a component of the National Institutes of Health (NIH), and NIH Roadmap for Medical Research. This grant did not present any conflict of interest with this manuscript.

\section{Conflicts of interest}

There are no conflicts of interest.

\section{RefERENCES}

1. Sihler KC, Napolitano LM. Complications of massive transfusion. Chest 2010;137:209-20.

2. Kleinman S, Caulfield T, Chan P, Davenport R, McFarland J, McPhedran S, et al. Toward an understanding of transfusion-related acute lung injury: Statement of a consensus panel. Transfusion 2004:44:1774-89.

3. Toy P, Popovsky MA, Abraham E, Ambruso DR, Holness LG, Kopko PM, et al. Transfusion-related acute lung injury: Definition and review. Crit Care Med 2005;33:721-6.

4. Benson AB, Austin GL, Berg M, McFann KK, Thomas S, Ramirez G, et al. Transfusion-related acute lung injury in ICU patients admitted with gastrointestinal bleeding. Intensive Care Med 2010;36:1710-7.

5. Gajic O, Rana R, Winters JL, Yilmaz M, Mendez JL, Rickman OB, et al. Transfusion-related acute lung injury in the critically ill: Prospective nested case-control study. Am J Respir Crit Care Med 2007;176:886-91.

6. Chaiwat O, Lang JD, Vavilala MS, Wang J, MacKenzie EJ, Jurkovich GJ, et al. Early packed red blood cell transfusion and acute respiratory distress syndrome after trauma. Anesthesiology 2009;110:351-60.

7. Gajic O, Rana R, Mendez JL, Rickman OB, Lymp JF, Hubmayr RD, et al. Acute lung injury after blood transfusion in mechanically ventilated patients. Transfusion 2004;44:1468-74.
8. Vlaar AP, Binnekade JM, Prins D, van Stein D, Hofstra JJ, Schultz MJ, et al. Risk factors and outcome of transfusion-related acute lung injury in the critically ill: A nested case-control study. Crit Care Med 2010;38:771-8.

9. Gajic O, Dara SI, Mendez JL, Adesanya AO, Festic E, Caples SM, et al. Ventilator-associated lung injury in patients without acute lung injury at the onset of mechanical ventilation. Crit Care Med 2004;32:1817-24.

10. Khan H, Belsher J, Yilmaz M, Afessa B, Winters JL, Moore SB, et al. Fresh-frozen plasma and platelet transfusions are associated with development of acute lung injury in critically ill medical patients. Chest 2007;131:1308-14.

11. Miller TE. New evidence in trauma resuscitation-is 1:1:1 the answer? Perioper Med (Lond) 2013;2:13.

12. McDaniel LM, Neal MD, Sperry JL, Alarcon LH, Forsythe RM, Triulzi $\mathrm{D}$, et al. Use of a massive transfusion protocol in nontrauma patients: Activate away. J Am Coll Surg 2013;216:1103-9.

13. Malone DL, Hess JR, Fingerhut A. Massive transfusion practices around the globe and a suggestion for a common massive transfusion protocol. J Trauma 2006;60 6 Suppl:S91-6.

14. Bernard GR, Artigas A, Brigham KL, Carlet J, Falke K, Hudson L, et al. The American-European Consensus Conference on ARDS. Definitions, mechanisms, relevant outcomes, and clinical trial coordination. Am J Respir Crit Care Med 1994;149:818-24.

15. Ellis RK. Determination of PO2 from saturation. J Appl Physiol 1989;67:902.

16. Severinghaus JW. Simple, accurate equations for human blood O2 dissociation computations. J Appl Physiol Respir Environ Exerc Physiol 1979;46:599-602.

17. Rice TW, Wheeler AP, Bernard GR, Hayden DL, Schoenfeld DA, Ware LB; National Institutes of Health, et al. Comparison of the $\mathrm{SpO}_{2} / \mathrm{FiO}_{2}$ ratio and the $\mathrm{PaO} 2 / \mathrm{FiO}_{2}$ ratio in patients with acute lung injury or ARDS. Chest 2007;132:410-7.

18. Pandharipande PP, Shintani AK, Hagerman HE, St. Jacques PJ, Rice TW, Sanders NW, et al. Derivation and validation of $\mathrm{SpO}_{2} / \mathrm{FiO}_{2}$ ratio to impute for $\mathrm{pao} 2 / \mathrm{FiO}_{2}$ ratio in the respiratory component of the sequential organ failure assessment score. Crit Care Med 2009;37:1317-21.

19. Brown SM, Grissom CK, Moss M, Rice TW, Schoenfeld D, Hou PC, et al. Nonlinear imputation of $\mathrm{Pao} 2 / \mathrm{FiO}_{2}$ from $\mathrm{SpO}_{2} / \mathrm{FiO}_{2}$ among patients with acute respiratory distress syndrome. Chest 2016;150:307-13.

20. Wiesner RH, McDiarmid SV, Kamath PS, Edwards EB, Malinchoc M, Kremers WK, et al. MELD and PELD: Application of survival models to liver allocation. Liver Transpl 2001;7:567-80.

21. Knaus WA, Draper EA, Wagner DP, Zimmerman JE. APACHE II: A severity of disease classification system. Crit Care Med 1985;13:818-29.

22. Afessa B, Gajic O, Keegan MT, Seferian EG, Hubmayr RD, Peters SG. Impact of introducing multiple evidence-based clinical practice protocols in a medical Intensive Care Unit: A retrospective cohort study. BMC Emerg Med 2007;7:10.

23. Rubenfeld GD, Angus DC, Pinsky MR, Curtis JR, Connors AF Jr., Bernard GR, et al. Outcomes research in critical care: Results of the American Thoracic Society Critical Care Assembly Workshop on Outcomes Research. The Members of the Outcomes Research Workshop. Am J Respir Crit Care Med 1999;160:358-67.

24. Fatalities Reported to FDAFollowing Blood Collection and Transfusion: Annual Summary for Fiscal Year 2012; 2014.

25. Toy P, Gajic O, Bacchetti P, Looney MR, Gropper MA, Hubmayr R, et al. Transfusion-related acute lung injury: Incidence and risk factors. Blood 2012;119:1757-67.

26. Kamath PS, Wiesner RH, Malinchoc M, Kremers W, Therneau TM, Kosberg CL, et al. A model to predict survival in patients with end-stage liver disease. Hepatology 2001;33:464-70.

27. Kim WR, Biggins SW, Kremers WK, Wiesner RH, Kamath PS, Benson JT, et al. Hyponatremia and mortality among patients on the liver-transplant waiting list. N Engl J Med 2008;359:1018-26.

28. Holcomb JB, Tilley BC, Baraniuk S, Fox EE, Wade CE, Podbielski JM, et al. Transfusion of plasma, platelets, and red blood cells in a 1:1:1 vs. a 1:1:2 ratio and mortality in patients with severe trauma: The PROPPR randomized clinical trial. JAMA 2015;313:471-82.

29. Tremblay A, Bandi V. Impact of body mass index on outcomes following critical care. Chest 2003;123:1202-7. 
30. Garrouste-Orgeas M, Troché G, Azoulay E, Caubel A, de Lassence A, Cheval C, et al. Body mass index. An additional prognostic factor in ICU patients. Intensive Care Med 2004;30:437-43.

31. Goulenok C, Monchi M, Chiche JD, Mira JP, Dhainaut JF, Cariou A, et al. Influence of overweight on ICU mortality: A prospective study. Chest 2004;125:1441-5.

32. Pickkers P, de Keizer N, Dusseljee J, Weerheijm D, van der Hoeven JG,
Peek N, et al. Body mass index is associated with hospital mortality in critically ill patients: An observational cohort study. Crit Care Med 2013;41:1878-83.

33. Akinnusi ME, Pineda LA, El Solh AA. Effect of obesity on intensive care morbidity and mortality: A meta-analysis. Crit Care Med 2008;36:151-8.

34. Narick C, Triulzi DJ, Yazer MH. Transfusion-associated circulatory overload after plasma transfusion. Transfusion 2012;52:160-5. 